\title{
CONNECTING A DISCRETE IONIC SIMULATION TO A CONTINUUM*
}

\author{
B. NADLER ${ }^{\dagger}$, T. NAEH ${ }^{\dagger}$, AND Z. SCHUSS $^{\dagger}$
}

\begin{abstract}
An important problem in simulating ions in solution is the connection of the finite simulation region to the surrounding continuum bath. In this paper we consider this connection for a simulation of uncharged independent Brownian particles and discuss the relevance of the results to a simulation of charged particles (ions). We consider a simulation region surrounded by a buffer embedded in a continuum bath. We analyze the time course of the exchange process of particles between the simulation region and the continuum, including re-entrances of particles that left the simulation. We partition the particle population into (i) those that have not yet visited the simulation and (ii) those that have. While the arrival process into the simulation of population (i) is Poissonian with known rate, that of population (ii) is more complex. We identify the ordered set of re-entrance times of population (ii) as a superposition of an infinite number of delayed terminating renewal processes, where the renewal periods may be infinite with positive probability. The ordered entrance times of populations (i) and (ii) form the pooled process of injection times of particles into the simulation. We show that while the pooled process is stationary, it is not Poissonian but rather has infinite memory. Yet, under some conditions on the sizes of the simulation and buffer regions, it can be approximated by a Poisson process. This seems to be the first result on the time course of a discrete simulation of a test volume embedded in a continuum.
\end{abstract}

Key words. stationary stochastic processes, diffusion, renewal theory, simulation of ions

AMS subject classifications. 60G10, 60G35, 60J60, 60K05

PII. S0036139901393688

1. Introduction. Computer simulations of ions in electrolytic solutions are a widely used tool in physical chemistry and are becoming increasingly important in molecular biophysics as well [1], [2], [3], [4], [5], [6], [7], [8], [9], [10], [11], [12], [13], [14], [15], [16]. Since it is impractical to simulate the entire continuum bath, a common approach is to isolate a small finite region of the continuum and simulate only the motion of ions located in this region. The requirements from such a "small" simulation are that the averaged concentrations of the different ionic species in the simulation volume be preserved, the electrostatic forces be correctly reproduced, and the effective measured ionic diffusion coefficients be recovered

Of course, as simulated ions may reach the boundary of the simulation region and nonsimulated bath ions may cross it, the simulation must be connected to the surrounding continuum bath. This involves not only the correct computation of the electrostatic field, including the contribution of nonsimulated bath ions, but also the resolution of the two following issues: (i) the imposed boundary behavior on trajectories of simulated ions as they reach the boundary of the simulation region and (ii) the injection scheme (if any) of new ions into the simulation. In this paper we are concerned with these two issues. Specifically, we study the processes of random exit,

${ }^{*}$ Received by the editors August 10, 2001; accepted for publication July 26, 2002; published electronically January 17, 2003.

http://www.siam.org/journals/siap/63-3/39368.html

$\dagger$ Department of Applied Mathematics, School of Mathematical Sciences, Tel-Aviv University, TelAviv 69978, Israel (boaz.nadler@yale.edu, galor@cs.cornell.edu, schuss@post.tau.ac.il). The research of the first author was partially supported by a research grant from DARPA. The research of the second author was partially supported by a grant from the Israel Ministry of Science and Technology. The research of the third author was partially supported by research grants from the US-Israel Binational Science Foundation, from the Israel Science Foundation, and from DARPA. 
entrance, and re-entrance of particles between the simulation region and the continuum bath. We analyze a simulation of uncharged particles and discuss the relevance of our results to a simulation with charged particles in section 8 . The computation of the electrostatic field for a simulation of charged particles will be considered elsewhere.

The total number of simulated ions in a simulation scheme can be either fixed or variable. In simulations with a fixed number of ions, there is no injection scheme of new ions into the simulation, and the imposed boundary conditions on the trajectories of simulated particles are either periodic or reflecting [15], [16]. Simulations with a fixed number of ions, and in particular those with periodic boundary conditions, have serious limitations which have been discussed at length in the literature [17], [18], [19], [20], [21], [22], [23] (and references therein). In particular, density fluctuations are absent in such simulations, and the computation of the electrostatic field is at best problematic.

Density fluctuations are determinants of important properties of an ionic solution [12], [13]. There have been various attempts in the literature to include density fluctuations in simulations with a fixed number of ions. The most common method is the introduction of a buffer region between the simulation region and the surrounding continuum bath. The simplest approach, as described in [6], is to run a simulation with a fixed total number of particles in the simulation and buffer region, with reflecting boundary conditions at the outer buffer boundary. In this scheme density fluctuations are of course present in the smaller simulation region, although it is unclear how faithfully they reproduce the actual density fluctuations in the simulation region. Other approaches, as reported in [2] and [8], replace the reflecting boundary conditions at the boundary of the buffer region by "soft" boundary conditions. That is, ions are allowed to leave the buffer region into the bath, but then they are subject to an artificial attracting force, so that they eventually return into the buffer region. In both references, the attracting force was designed to maintain the correct equilibrium density in the simulation region. Once again, while the total number of particles is kept fixed, there are fluctuations in the number of particles in the smaller simulation region. The main problem with these approaches is that the confinement of ions to the simulation by ad hoc artificial attracting forces (or even infinite forces, in the case of reflecting boundaries) imposes unphysical conditions on the simulation and may not necessarily lead to correct time dependent density fluctuations.

Simulations with a variable number of ions also use a buffer region between the simulation and the continuum bath, but replace the reflecting or soft boundary conditions at the boundary of the buffer region by stochastic boundary conditions [7], [8], [9], [10]. These conditions introduce a random exchange mechanism of ions between the simulation and buffer regions with the aim of reproducing the equilibrium density fluctuations. Obviously, different assumptions on the stochastic boundaries lead to different density fluctuations in time and space inside the simulation region. Unfortunately, the stochastic process of equilibrium density fluctuations is unknown in the sense that the joint probability distribution of the number of particles in the simulation volume at different times is unknown. The fluctuation theory proposed by Smoluchowski [11] is valid only for sufficiently long time intervals between observations so that it cannot be applied to a simulation of particles in solution [29]. Yet, these fluctuations affect the physical properties of the solute [12], so proposing a scheme that recovers the correct fluctuations is essential.

In all formulations of stochastic boundaries, the probability laws for the injection times of new particles are assumed, rather than derived, from the laws of motion of 
ions in solution. The aim of this paper is to derive the probability laws of the entrance and re-entrance processes of ions into a finite volume surrounded by a buffer zone as they actually occur in the solution. To derive our results, we make standard general assumptions of physical chemistry about the ionic motion of bath ions.

In our analysis, we consider a finite simulation region surrounded by a buffer region embedded in a practically infinite ionic solution. We assume that all bath ions can be described as independent uncharged Brownian particles with an effective diffusion coefficient. This assumption is commonly used in physical chemistry, where ionic solutions are described by an electrochemical potential [12]. This means that, on a large enough time scale, the motions of charged interacting ions in the bath are assumed independent noninteracting diffusion processes in a mean field, which reduce to independent Brownian particles for a vanishing mean field.

We consider particle entrances at the boundary of the inner region and their exits at the outer boundary of the buffer zone. In the corresponding simulation, the motion of all particles that enter the inner region is simulated until they cross the outer boundary of the buffer region for the first time. Their motion is simulated once again the next time they enter the inner region, until their next exit at the outer region, and so on. The buffer region in the simulation scheme serves as a separator between the inner simulation region and the surrounding continuum bath, thus avoiding instantaneous re-entrances of Brownian particles at the boundary of the inner region,

To formulate mathematically the problem of introducing particles into the simulation, we divide their entrances into two types: (i) arrivals of "new" particles, which have not visited the simulation region so far, and (ii) arrivals of "returning" particles, which have already visited and exited the simulation region. Obviously, the probability law of the recirculation times is different from that of the times between new arrivals, so that particles that leave the simulation at the outer boundary of the buffer zone cannot be returned to the bath on equal footing with particles that have not been in the simulation so far.

In our previous paper [24], we studied the stationary arrival process (i) of new particles. It was shown that in steady state, the interarrival times to an absorbing boundary are exponentially distributed with rate equal to the Smoluchowski flux, rendering the stationary arrival process Poissonian. Apart from its relevance to the problem of connecting a simulation to the surrounding continuum, the study of the arrival problem at an absorbing boundary has many physical applications and a long mathematical history [25], [26].

In this paper, we study the recirculation process (ii) and its role in connecting the simulation to the surrounding bath. We determine stationary probability laws governing the entrance and re-entrance times of all processes (i) and (ii). At any given time in the course of the simulation the particle to be injected next is the one whose arrival time at the inner sphere is the shortest among all the particles not currently in the simulation. The candidates for injection are both the new and recirculating particles. In this paper we identify the injection process as a pooled process, that is, a superposition of an infinite number of terminating renewal processes and determine some of its statistical properties.

We show that the pooled process converges to a stationary steady state. However, even though in the steady state the process is stationary, its interarrival times are not exponential, not even independent, and have infinite memory. We calculate some of the statistical properties of the pooled process, such as the exact pdf of the residual 
first arrival time of the pooled process in steady state, as well as the first and second moments of the pooled process. Our main result is that under some conditions on the size of the simulation and buffer regions, the infinite memory pooled process can be approximated by a Poisson process. This approximation considerably simplifies the simulation. To the best of our knowledge, this work seems to be the first result on the time course of a discrete simulation embedded in a continuum.

The paper is organized as follows. In section 2 , we formulate the simulation scheme and identify the entrance process of particles as a pooled process. In section 3 , we present a continuum model of the simulation, from which the average flux of the pooled process is calculated. The first two moments of the pooled process are calculated in section 4 by renewal-type considerations. In section 5 , we define the entrance times of the pooled process, and in section 6 , we calculate the distribution of the residual time till the first particle entrance and the distribution of the subsequent interarrival time. The main result, which asserts that short interarrival times are exponentially distributed and the effective exponential rate is the same as that calculated from the continuum and renewal models, is discussed in section 7 . We also present there results of a simulation of the pooled process and discuss its rate of convergence to steady state. Section 8 contains a summary and discussion.

2. Setup of the problem. We consider the following simulation scheme: A practically infinite ionic bath of average density $\rho$ occupies the three dimensional space. Inside this bath, there is a finite simulation region consisting of two concentric spheres of radii $a$ and $r_{0}\left(a<r_{0}\right)$, centered at the origin (see Figure 1(a)). In the proposed simulation scheme, the motion of all particles that enter the inner sphere is simulated until they cross the outer sphere for the first time. Their motion is simulated once again the next time they enter the inner sphere, until their next exit at the outer sphere, and so on (see Figure 1(b)). The region beyond the outer sphere, $|\boldsymbol{r}|>r_{0}$, contains no simulated particles and is described by a continuum particle density. The annular ring $a<|\boldsymbol{r}|<r_{0}$ is a buffer region that connects the inner region to the surrounding continuum bath in $|\boldsymbol{r}|>r_{0}$. The buffer region is part continuum and part discrete in the sense that the motions of only some of the particles in it are simulated.

While we do not describe the exact electrostatic interactions between bath ions, we follow the common practice in chemical physics [12] that describes the effective motions of the nonsimulated bath ions as independent diffusions in a mean field. Specifically, for a homogeneous bath with no applied potential, the mean field vanishes, so that exterior of the simulation region can be described as an infinite bath of independent free Brownian particles, with average density $\rho$. As discussed in the introduction, we assume that the simulation is self-consistent. This means that on a large enough time scale the coarse grained motion of simulated ions inside the simulation region can also be described as free diffusion with the same diffusion coefficient as that assumed for the nonsimulated ions in the continuum bath. We further assume that the simulation and buffer regions are large enough so that, for the purpose of calculating the time course of the simulation, all particles, both simulated and nonsimulated, can be described as effectively independent Brownian particles with the above diffusion coefficient. We note that the self-consistency condition is not trivial, and it determines important physical parameters, as discussed in [27].

We introduce the following notation. Particles that have not visited the inner sphere so far are called blue particles, and those that have are called red particles. The arrival process of blue particles into the simulation is process (i) and the re- 


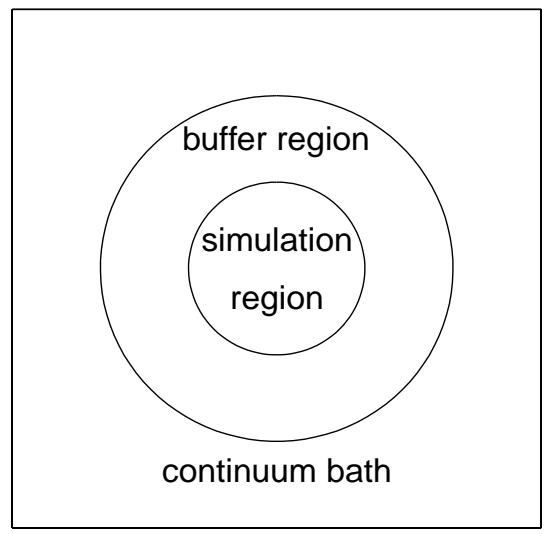

(a)

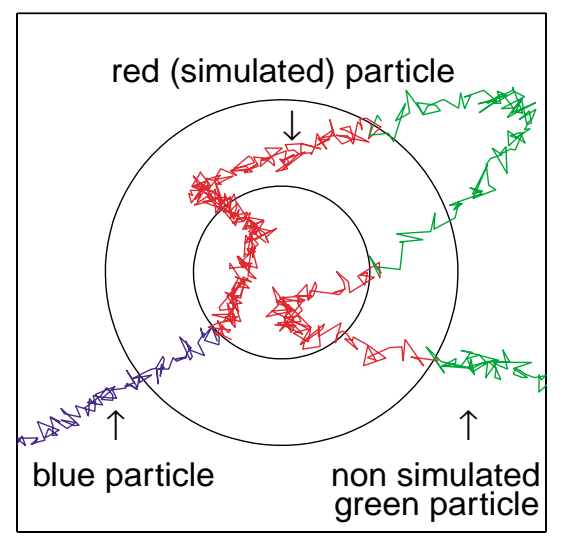

(b)

FIG. 1. (a) The simulation setup. (b) The simulated and nonsimulated parts of a typical particle trajectory.

entrance of the red particles is process (ii).

As shown in [24], in steady state, blue Brownian particles arrive at an absorbing sphere at an exponential rate. A blue particle that reaches the boundary of the inner sphere turns red instantaneously and stays red forever. As long as it is inside the simulation region, its dynamics change from independent Brownian motion, with its effective diffusion coefficient in the ambient solution, to diffusive motion governed by the Langevin equation with electrostatic interactions with the ions in the simulation and with the far field of the ambient solution. The interactions with the nonsimulated ions in the ambient solution outside the outer sphere are replaced by interactions with a mean field, as mentioned above. The assumption of a self-consistent simulation implies that for our purposes the probability distribution of the time a simulated ion spends inside the simulation is identical to that of a free noninteracting Brownian particle.

One way to run this simulation is as follows: Introduce blue particles at exponential interarrival times. Follow the trajectory of each (now red) simulated particle until its first exit at the boundary of the larger sphere. Now, sample a random reentrance time into the simulation, assuming it performs Brownian motion outside the inner sphere, and store this re-entrance time in a table of all re-entrance times of recirculating particles. In this scheme, the next particle to be injected into the simulation is the one with the minimal return time between all particles registered in the table and the next blue particle to be injected into the simulation. There are two main difficulties with this simulation scheme. One is that the table of re-entrance times grows indefinitely with time, because the mean recirculation time of returning particles is infinite (see Proposition A.1 in Appendix A). The other difficulty is that the convergence to steady state of this simulation is extremely slow, as analyzed in section 7 . This is due to the fact that as long as the table is finite, all the infinite number of re-entrances of particles that were inside the simulation region before the simulation actually started, and are thus not present in this table, are neglected.

This simple example shows that a mechanism to run the simulation in steady state from its start needs to be developed. More specifically, the steady state distribution of return times from this infinite table of recirculated particles has to be calculated. 
A key point in this calculation is the well-known feature [28] that for free Brownian motion in three dimensions there is a positive probability that a red particle that just exited the simulation will never return to the inner sphere, so that its recirculation time is infinite. This observation gives rise to the following renewal-type model. The arrivals of blue particles at the inner sphere form a Poisson process [24], [29], as mentioned above. For each arriving particle, its subsequent re-entrance times into the simulation form an independent renewal process. The interarrival times of this process may be infinite with positive probability, thus rendering it a terminating renewal process [30]. The renewal processes of different particles start of course at different times, according to their first injection times. A renewal process that starts at a random time with one distribution and is renewed with another is called a delayed renewal process [30]. The superposition of all the delayed renewal processes is called the pooled process. The steady state of the pooled process is the process of introducing new particles into the simulation, which is the concern of this paper.

3. A continuum model of the simulation. In this section we compute the average flux of particle entrances of the pooled process (both blue and red) into the inner simulation region from a continuum model of the above described simulation. To this end, we represent the Brownian particles in the simulation and in the bath as continuum densities. Since we consider free Brownian particles, all densities are spherically symmetric and depend only on the radial distance, $r=|\boldsymbol{r}|$, from the center of the simulation spheres. The densities of simulated particles are defined as averages of many different realizations of particle locations of a running simulation.

We start from the radial density of the blue particles, denoted $p_{B}(r)$. It satisfies the diffusion equation outside the inner sphere [31],

$$
\Delta p_{B}(r)=\frac{d^{2} p_{B}(r)}{d r^{2}}+\frac{2}{r} \frac{d p_{B}(r)}{d r}=0 \quad \text { for } r>a,
$$

with absorbing boundary conditions at the boundary of the inner sphere, where blue particles instantaneously turn red,

$$
p_{B}(a)=0 .
$$

In addition, far away from the simulation region the blue particle density equals the bulk density $\rho$,

$$
\lim _{r \rightarrow \infty} p_{B}(r)=\rho
$$

The solution of (3.1)-(3.3) is

$$
p_{B}(r)= \begin{cases}\rho\left(1-\frac{a}{r}\right) & \text { for } \quad r>a, \\ 0 & \text { for } \quad r<a .\end{cases}
$$

Using (3.4), the continuum flux of blue particles at the inner sphere is given by

$$
J_{\text {blue }}=-\left.4 \pi a^{2} D \frac{d}{d r} p_{B}(r)\right|_{r=a}=4 \pi \rho a D
$$

where $\rho$ is the bulk concentration at infinity, and $D$ is the diffusion coefficient of bath particles. Equation (3.5) for the average flux of Brownian particles at an absorbing boundary was already calculated by Smoluchowski in 1917 [25]. 
Next, we consider the red particle density. According to our assumptions, the total particle density in the bath is uniform and at all locations equals the bulk density $\rho$. Since the simulation region is an arbitrary region of the bath, the steady state density of the red particles, denoted $p_{R}(r)$, complements that of the blue particles to the bulk density $\rho$,

$$
p_{R}(r)=\rho-p_{B}(r) .
$$

We denote by $J_{\text {total }}$ the total flux of particle entrances at the inner sphere. The total flux is the sum of the blue particles flux given by (3.5), and the flux of returning red particles, which have exited the simulation through the outer sphere. Since at any given time, only some of the red particles are simulated while others are not, the flux of returning red particles cannot be computed from (3.6). The contribution of red particles to the total incoming flux at the inner sphere comes only from the nonsimulated population of red particles. To compute their contribution to the influx, we divide the red particle population into two: simulated red particles, denoted pink particles, and nonsimulated red particles, denoted green particles. With this notation, the total flux at the inner sphere is given by

$$
J_{\text {total }}=J_{\text {blue }}+J_{\text {green }}
$$

We denote the steady state densities of the pink and green particles by $p_{P}(r)$ and $p_{G}(r)$, respectively. Of course,

$$
p_{R}(r)=p_{P}(r)+p_{G}(r) .
$$

We now calculate the densities of the green and pink particles. Each particle that enters the simulation region at the inner sphere, either new or returning particle, exits it at the outer sphere at some later time with probability one. Once such a particle crosses the outer sphere it immediately becomes green, until its next arrival at the inner sphere, when it becomes pink again. Thus, the green particle density has a source at the outer sphere whose strength equals the yet undetermined total absorption flux $J_{\text {total }}$ of both blue and returning red particles. That is, the green particle density satisfies the diffusion equation

$$
\Delta p_{G}(r)=\frac{d^{2} p_{G}(r)}{d r^{2}}+\frac{2}{r} \frac{d p_{G}(r)}{d r}=J_{\text {total }} \frac{\delta\left(r-r_{0}\right)}{4 \pi r_{0}^{2}}, \quad \text { for } \quad r>a,
$$

with absorbing boundary conditions at the inner sphere,

$$
p_{G}(a)=0,
$$

and with the condition that $p_{G}(r) \rightarrow 0$ as $r \rightarrow \infty$. The solution for the green particle density, in terms of the yet undetermined parameter $J_{\text {total }}$, is given by

$$
p_{G}(r)= \begin{cases}\frac{J_{\text {total }}}{4 \pi r_{0}}\left[\left(1-\frac{a}{r}\right)-H\left(r-r_{0}\right)\left(1-\frac{r_{0}}{r}\right)\right], & \text { for } \quad r>a, \\ 0, & \text { for } r<a,\end{cases}
$$

where $H(x)$ denotes the Heaviside step function. The averaged densities of the blue, pink, and green particle populations are shown in Figure 2. 


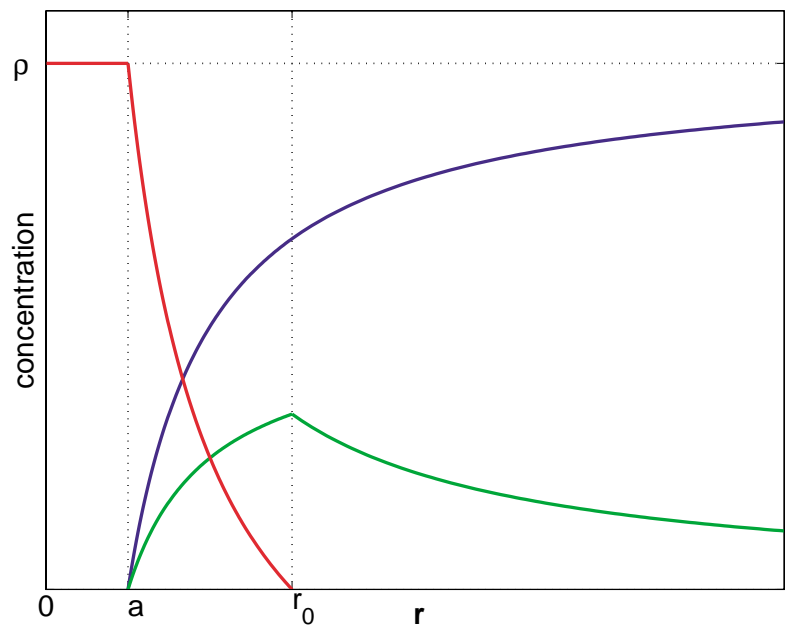

FIG. 2. The averaged densities of the blue, pink, and green particle population.

The average influx of green particles at the inner sphere, denoted $J_{\text {green }}$, is thus

$$
J_{\text {green }}=\left.4 \pi r^{2} \frac{d}{d r} p_{G}(r)\right|_{r=a}=\frac{a}{r_{0}} J_{\text {total }} .
$$

Note that the flux of green particles into the inner sphere is smaller than its source strength $J_{\text {total }}$. This is because there is a positive probability for a green particle that starts its motion at the boundary of the outer sphere to never reach the inner sphere. The ratio between the two fluxes is simply the probability of a free Brownian particle to ever reach the inner sphere from the outer sphere. As shown in section 6 , this return probability, denoted $p$, is given by

$$
p=\frac{a}{r_{0}} .
$$

The total mean flux of particles into the simulation, denoted $\Lambda$, can now be obtained by combining (3.5), (3.7), and (3.10),

$$
\Lambda=J_{\text {total }}=J_{\text {blue }}+J_{\text {green }}=\frac{\lambda_{B}}{1-p} .
$$

As expected, due to the recirculating red particles, the total flux at the inner sphere is larger than the flux of only the blue particles. For example, in a simulation scheme that inserts only blue particles, absorbs them at the outer sphere and "forgets" about their possible re-entrances, the average particle flux into the simulation region is smaller than it should be. This might have serious effects on the outcome of the simulation.

Finally, note that the flux parameter $\Lambda$ does not represent a physical quantity, but is rather only a simulation parameter, that depends on the choice of the radii $a$ and $r_{0}$ of the simulation spheres. Therefore, all physical parameters that are an outcome of the simulation must not depend on $\Lambda$.

4. The mean and variance of the pooled process. In the previous section the total average influx of the steady state pooled process was computed with the 
aid of a corresponding continuum model. However, the continuum model is unable to compute other statistical properties of the pooled process, such as the distribution of the interarrival times, or even their variance. Obviously, a simulation scheme should attempt to preserve at least some of these quantities. In this section, we present a statistical renewal model of the pooled process that, in principle, enables the computation of all moments of the pooled process.

For simplicity, we consider a simulation that starts at time $t=0$ with no particles initially inside the simulation region. Since we are interested in quantities for a simulation that has reached steady state, the results are independent of these initial conditions. We denote by $N^{P}(t)$ the total number of particle entrances of the pooled process by time $t$. This includes all entrances of blue particles and re-entrances of returning red particles into the simulation region by time $t$. We compute the first two moments of $N^{P}(t)$ and note that the method presented can be applied to compute all higher order moments as well.

THEOREM 4.1. The average steady state flux of the pooled process is $\Lambda$, and its variance per unit time is $\Lambda(1+p) /(1-p)$.

Proof. Let $N^{B}(t)$ denote the number of arrivals of blue particles into the simulation during the time interval $[0, t]$, and let $\left\{t^{i}\right\}_{i=1}^{N^{B}(t)}$ denote these arrival times. According to [24], the interarrival times of the blue particles are independent identically distributed (i.i.d.) random variables, exponentially distributed with rate $\lambda_{B}$ that is equal to the corresponding continuum flux $J_{\text {blue }}$ calculated in (3.5). Therefore, the total number of blue arrivals by time $t$, denoted $N^{B}(t)$, is a Poisson distributed random variable with parameter $\lambda_{B} t$. For each blue particle we denote by $\xi\left(t-t^{i}\right)$ the (random) number of its re-entrances into the simulation by time $t$ since its first entrance at time $t^{i}$. In terms of these random variables, the total number of particle entrances of the pooled process can be written as

$$
N^{P}(t)=\sum_{i=1}^{N^{B}(t)}\left[1+\xi\left(t-t^{i}\right)\right] .
$$

We denote by $\mu_{1}(t)$ and $\mu_{2}(t)$ the first two moments of $\xi(t)$,

$$
\mu_{1}(t)=E[\xi(t)], \quad \mu_{2}(t)=E[\xi(t)]^{2} .
$$

Note that $\xi(\infty)$ is the total number of re-entrances of a red particle. Since each particle has probability $p<1$ of ever returning to the simulation (see (3.11)), the random variable $\xi(\infty)$ follows a geometric distribution with parameter $p$, which gives

$$
\mu_{1}(\infty)=\frac{p}{1-p}, \quad \mu_{2}(\infty)=\frac{p}{1-p}+\frac{2 p^{2}}{(1-p)^{2}} .
$$

To compute the average of the pooled process, $E\left[N^{P}(t)\right]$, we divide the sum in (4.1) into the first term and the sum of the $N^{B}(t)-1$ remaining terms,

$$
N^{P}(t)=1+\xi\left(t-t^{1}\right)+\sum_{i=2}^{N^{B}(t)}\left(1+\xi\left(t-t^{i}\right)\right) .
$$

According to our assumptions, the arrival process of blue particles is Poissonian and thus memoryless. In addition, the recirculation processes of different particles are independent and identical, if their starting times are all shifted to an identical initial 
time. These two properties imply that given the arrival time of the first blue particle, $t^{1}=s$, the sum in (4.4) has the same statistical properties as that of the random variable $N^{P}(t-s)$. In particular,

$$
\begin{aligned}
\operatorname{Pr}\left\{\sum_{i=2}^{N^{B}(t)}\left(1+\xi\left(t-t^{i}\right)\right)=n \mid t^{1}=s\right\} & =\operatorname{Pr}\left\{\sum_{i=2}^{N^{B}(t)}\left[1+\xi\left((t-s)-\left(t^{i}-s\right)\right)\right]=n\right\} \\
& =\operatorname{Pr}\left\{N^{P}(t-s)=n\right\} .
\end{aligned}
$$

Thus, taking expectations in (4.4) and using the exponential distribution of $t^{1}$ gives

$$
\begin{aligned}
E\left[N^{P}(t)\right] & =\int_{0}^{t} E\left[N^{P}(t) \mid t^{1}=s\right] \lambda_{B} e^{-\lambda_{B} s} d s \\
& =\int_{0}^{t}\left[1+\mu_{1}(t-s)+E \sum_{i=2}^{N^{B}(t)}\left[1+\xi\left((t-s)-\left(t^{i}-s\right)\right)\right]\right] \lambda_{B} e^{-\lambda_{B} s} d s,
\end{aligned}
$$

which, according to (4.5), can equivalently be written as a renewal-type integral equation,

$$
E\left[N^{P}(t)\right]=\int_{0}^{t} \lambda_{B} e^{-\lambda_{B} s}\left\{1+\mu_{1}(t-s)+E\left[N^{P}(t-s)\right]\right\} d s
$$

along with the initial condition $N^{P}(0)=0$. The solution of (4.6) is given by

$$
E\left[N^{P}(t)\right]=\int_{0}^{t} \lambda_{B}\left[1+\mu_{1}(s)\right] d s .
$$

Therefore, by l'Hôpital's rule and using (4.3),

$$
\lim _{t \rightarrow \infty} \frac{E\left[N^{P}(t)\right]}{t}=\lim _{t \rightarrow \infty} \lambda_{B}\left[1+\mu_{1}(t)\right]=\lambda_{B}\left[1+\mu_{1}(\infty)\right]=\Lambda .
$$

As expected, we recover the same total average flux of the pooled process as that computed from the continuum model, (3.12).

Next, we consider the second moment, $E\left[N^{P}(t)^{2}\right]$. Before computing the expectation, we write $\left[N^{P}(t)\right]^{2}$ as

$$
\begin{aligned}
{\left[N^{P}(t)\right]^{2} } & =\sum_{i=1}^{N^{B}(t)}\left[1+2 \xi\left(t-t^{i}\right)+\xi^{2}\left(t-t^{i}\right)\right]+2 \sum_{i=1}^{N^{B}(t)}\left[1+\xi\left(t-t^{i}\right)\right] \sum_{j=i+1}^{N^{B}(t)}\left[1+\xi\left(t-t^{j}\right)\right] \\
& =H(t)+2 G(t),
\end{aligned}
$$

where

$$
\begin{aligned}
& H(t)=\sum_{i=1}^{N^{B}(t)}\left[1+2 \xi\left(t-t^{i}\right)+\xi^{2}\left(t-t^{i}\right)\right] \\
& G(t)=\sum_{i=1}^{N^{B}(t)}\left[1+\xi\left(t-t^{i}\right)\right] \sum_{j=i+1}^{N^{B}(t)}\left[1+\xi\left(t-t^{j}\right)\right] .
\end{aligned}
$$


The expectations of $H(t)$ and $G(t)$ also satisfy integral equations,

$$
\begin{aligned}
& E[H(t)]=\int_{0}^{t} \lambda_{B} e^{-\lambda_{B} s}\left\{1+2 \mu_{1}(t-s)+\mu_{2}(t-s)+E[H(t-s)]\right\} d s, \\
& E[G(t)]=\int_{0}^{t} \lambda_{B} e^{-\lambda_{B} s}\left\{\left(1+\mu_{1}(t-s)\right) E\left[N^{P}(t-s)\right]+E[G(t-s)]\right\} d s .
\end{aligned}
$$

The solutions of these equations are

$$
\begin{aligned}
& E[H(t)]=\int_{0}^{t} \lambda_{B}\left[1+2 \mu_{1}(s)+\mu_{2}(s)\right] d s, \\
& E[G(t)]=\int_{0}^{t} \lambda_{B}\left[1+\mu_{1}(s)\right] E\left[N^{P}(s)\right] d s .
\end{aligned}
$$

The long time behavior of the variance of $N^{P}(t)$ is found from the identity

$$
\begin{aligned}
\lim _{t \rightarrow \infty} \frac{\operatorname{Var}\left[N^{P}(t)\right]}{t} & =\lim _{t \rightarrow \infty} \frac{E\left\{\left[N^{P}(t)\right]^{2}\right\}-\left\{E N^{P}(t)\right\}^{2}}{t} \\
& =\lim _{t \rightarrow \infty} \frac{E[H(t)]+2 E[G(t)]-\left\{E\left[N^{P}(t)\right]\right\}^{2}}{t} .
\end{aligned}
$$

Inserting the expressions for all quantities, and applying l'Hôpital's rule, gives

$$
\lim _{t \rightarrow \infty} \frac{\operatorname{Var}\left[N^{P}(t)\right]}{t}=\Lambda\left[1+2\left(\frac{p}{1-p}\right)\right]=\Lambda \frac{1+p}{1-p} .
$$

Note that all moments of the pooled process are independent of the exact distribution of the recirculation time. Rather, they depend only on the return probability $p$.

Equation (4.8) clearly shows that the pooled process $N^{P}(t)$ is not Poissonian, since the variance per unit time of a Poisson process equals its average rate $\Lambda$. The variance of $N^{P}(t)$ is larger by a factor $(1+p) /(1-p)$, a phenomenon due to the possible re-entrances of exiting particles. In approximating the pooled process by a Poisson process with the same rate, this factor is lost.

5. The entrance times of the pooled process. In the previous section we calculated the first two moments of the pooled process. We now study the actual distribution of the interarrival times of the pooled process, that is, the PDF of the time between the consecutive introductions of particles into the simulation. As discussed above, these entrance times are the ordered union of both entrance times of new blue particles and re-entrance times of red recirculating particles.

Recall that the consecutive arrival times of blue particles were denoted $t^{j}$. We denote their interarrival times by $\tau^{j}=t^{j}-t^{j-1}$ with the convention that $t^{0}=0$. As shown in [24], the arrival process of blue particles is Poissonian with rate $\lambda_{B}$ given by (3.5). Therefore, $\tau^{j}$ are i.i.d. exponential random variables with a common PDF

$$
\operatorname{Pr}\left\{\tau^{j} \leq t\right\}=F_{B}(t)=1-\exp \left(-\lambda_{B} t\right) .
$$

Next, we consider the recirculation process of red particles. We introduce the following notation for the successive exit and re-entrance times of a red particle. The first entrance time to the inner sphere is denoted by $t_{1}^{j} \equiv t^{j}$. Its first exit time from 
the simulation at the outer sphere is denoted $\theta_{1}^{j}$, its next re-entrance time $t_{2}^{j}$, and so on. Thus $t_{1}^{j}<\theta_{1}^{j}<t_{2}^{j}<\theta_{2}^{j}<\ldots$.

The times $t_{n}^{j}$ are the consecutive re-entrance times of the $j$ th particle into the simulation. We denote $T_{1}^{j}=t_{1}^{j}$ and set $T_{n}^{j}=t_{n}^{j}-t_{n-1}^{j}$ for $n>1$. The times $T_{n}^{j}$ for $n>1$ are called the recirculation times of the $j$ th particle. According to our assumptions, these times are i.i.d. random variables with a positive probability to be infinite. We denote their PDF by

$$
F_{T}(t)=\operatorname{Pr}\left\{T_{n}^{j} \leq t\right\} .
$$

Their pdf is given by

$$
f_{T}(t)=f_{\tau_{\text {in }}} * f_{\tau_{\text {out }}}(t),
$$

where $\tau_{\text {in }}$ is the time a simulated (pink) particle spends in the simulation and $\tau_{\text {out }}$ is the time a nonsimulated (green) particle spends outside the simulation.

The assumption that the green particles have a positive probability $1-p$ of never returning from the outer sphere to the inner sphere is expressed as

$$
\lim _{t \rightarrow \infty} F_{T}(t)=p<1,
$$

or, equivalently,

$$
\operatorname{Pr}\left\{T_{n}^{j}=\infty\right\}=1-p>0 \quad(n>1),
$$

where $p$ is given by (3.11).

Recall that $\xi\left(t-t^{j}\right)$ denoted the number of re-entrances of the $j$ th red particle into the simulation by time $t$. We denote by $N^{j}(t)$ its total number of entrances into the simulation by time $t$, including the first entrance at time $t^{j}$,

$$
N^{j}(t)=1+\xi\left(t-t^{j}\right)
$$

Note that (5.3) implies that $N^{j}(t)$ is a terminating renewal process [30], that is, a renewal process that terminates when an infinite recirculation time occurs.

We now consider the entrance times of the pooled process. By definition, the total number of particle entrances into the simulation by time $t$, denoted $N^{P}(t)$, is given by

$$
N^{P}(t)=\sum_{j=1}^{N^{B}(t)} N^{j}(t) .
$$

The actual entrance times of the pooled process into the simulation, $\left\{S_{\ell}^{P}\right\}_{\ell=1}^{N^{P}(t)}$, are the elements of the set

$$
\left\{S_{\ell}^{P}\right\}_{\ell=1}^{N^{P}(t)}=\left\{t_{k}^{j} \mid 1 \leq j \leq N^{B}(t), 1 \leq k \leq N^{j}(t)\right\}
$$

arranged in ascending order.

The times between successive arrivals of the pooled process at the inner sphere, denoted $T_{\ell}^{P}$, are defined by

$$
T_{\ell}^{P}=S_{\ell+1}^{P}-S_{\ell}^{P}
$$

With this notation the mathematical problem of simulating the arrivals of ions at the inner sphere is to determine the joint PDF of the times $T_{\ell}^{P}$ (for all $\ell$ ). These times are the interarrival times for introducing new particles into the simulation. 
6. The first arrival time of the pooled process. In this section, we calculate the exact PDF of the first arrival time of the pooled process in the steady state. First, we compute the distribution of the residual time since start of observation of the simulation till the introduction of the first new particle into the inner sphere. Then, we compute the PDF of the time between consecutive arrivals of the pooled process.

We introduce the following notation. We denote by $\varphi(t)$ the renewal function of the recirculation process of a single particle. It is given by

$$
\varphi(t)=\sum_{k=0}^{\infty} f_{T}^{* k}(t),
$$

where $f_{T}^{* k}(t)$ is the $k$-convolution of the pdf $f_{T}(t)$ of a single recirculation time, and $f_{T}^{* 0}(t)=\delta(t)$. For future uses, we note that the Laplace transform of $\varphi$ is

$$
\widehat{\varphi}(s)=\sum_{k=0}^{\infty} \widehat{f_{T}^{* k}}(s)=\sum_{k=0}^{\infty}\left[\widehat{f}_{T}(s)\right]^{k}=\frac{1}{1-\widehat{f}_{T}(s)},
$$

and at $s=0$ we obtain from $(5.2)$

$$
\widehat{\varphi}(0)=\int_{0}^{\infty} \varphi(t) d t=\frac{1}{1-p} .
$$

Consider a simulation that has been running for an infinite time and is already in steady state. We start to observe the simulation at time $t=0$, and denote by $\gamma^{P}$ the first arrival time of a particle into the simulation after $t=0$. The first particle to arrive into the simulation may be either a blue particle that has not yet been in the simulation, or a red particle that has visited the simulation in the past and may re-enter the simulation region at the inner sphere after start of observation. Before we compute the exact PDF of $\gamma^{P}$, it is useful to compute the probability that a red particle that initially entered the simulation region at time $-s$ in the past will re-enter the simulation region at time $x$ after start of observation.

Lemma 6.1. Let $\gamma_{s}^{R}$ denote the first re-entrance time after $t=0$ of a red particle that initially entered the simulation at time $-s$. Then

$$
\operatorname{Pr}\left\{\gamma_{s}^{R}=x\right\}=\int_{0}^{s} \varphi(s-u) f_{T}(x+u) d u .
$$

Proof. Consider a blue particle that entered the simulation at time $t_{1}=-s$. In the time interval $[-s, 0]$ this particle may have re-entered the simulation an arbitrary number of times. For a particle that recursed $k-1$ times before time $t=0$, we denote by $t_{k}$ its last re-entrance time before $t=0$ and by $t_{k+1}$ its next re-entrance time after $t=0$. The event $\left\{\gamma_{s}^{R}=x\right\}$ can thus be decomposed into the disjoint union

$$
\left\{\gamma_{s}^{R}=x\right\}=\bigcup_{k=1}^{\infty}\left\{t_{k}<0 \cap t_{k+1}=x \mid t_{1}=-s\right\}
$$

so that

$$
\operatorname{Pr}\left\{\gamma_{s}^{R}=x\right\}=\sum_{k=1}^{\infty} \operatorname{Pr}\left\{t_{k}<0 \cap t_{k+1}=x \mid t_{1}=-s\right\} .
$$


The first summand is the probability that the first re-entrance time of the particle occurred at time $x$. Therefore,

$$
\operatorname{Pr}\left\{t_{1}<0 \cap t_{2}=x \mid t_{1}=-s\right\}=f_{T}(s+x) .
$$

The next summand $(k=2)$ is the probability of exactly one recirculation before time $t=0$ and next re-entrance at time $x$. To compute this probability, we integrate over all possible times $-u$ for the recirculation time $t_{2}$,

$$
\begin{aligned}
\operatorname{Pr}\left\{t_{2}<0 \cap t_{3}=x \mid t_{1}=-s\right\} & =\int_{0}^{s} \operatorname{Pr}\left\{t_{2}=-u \cap t_{3}=x \mid t_{1}=-s\right\} d u \\
& =\int_{0}^{s} f_{T}(s-u) f_{T}(u+x) d u .
\end{aligned}
$$

We now consider the $k$ th term in the sum (6.5). It represents the probability of exactly $k-1$ recirculations before time $t=0$ and next re-entrance at time $x$. Let $-u$ denote the last recirculation time prior to time $t=0$. By assumption, all recirculation times of a particle are i.i.d. random variables with pdf $f_{T}(t)$. Therefore,

$$
\operatorname{Pr}\left\{t_{k}=-u \mid t_{1}=-s\right\}=f_{T}^{*(k-1)}(s-u),
$$

where $f_{T}^{* k}$ denotes the $k$ th convolution of the pdf $f_{T}(t)$. Thus,

$$
\operatorname{Pr}\left\{t_{k}<0 \cap t_{k+1}=x \mid t_{1}=-s\right\}=\int_{0}^{s} f_{T}^{*(k-1)}(s-u) f_{T}(u+x) d u .
$$

Combining (6.5) with (6.6) and (6.8) and using the definition $f_{T}^{* 0}(t)=\delta(t)$, we obtain that

$$
\begin{aligned}
\operatorname{Pr}\left\{\gamma_{s}^{R}=x\right\} & =\int_{0}^{s} \sum_{k=0}^{\infty} f_{T}^{* k}(s-u) f_{T}(u+x) d u \\
& =\int_{0}^{s} \varphi(s-u) f_{T}(u+x) d u,
\end{aligned}
$$

which concludes the proof of the lemma.

We are now ready to prove the following theorem concerning the PDF of the first arrival time of the pooled process.

THEOREM 6.2. The stationary PDF of the first arrival time of a steady state pooled process is given by

$$
\operatorname{Pr}\left\{\gamma^{P}>x\right\}=\exp \{-\Lambda x\} \exp \left\{\Lambda \int_{0}^{x} F_{T}(t) d t\right\} .
$$

Proof. Consider a simulation that has been running for an infinite time which we start to observe at time $t=0$. The event $\left\{x<\gamma^{P}<x+\Delta x\right\}$ means that no particles arrived into the simulation in the time interval $[0, x]$ and exactly one particle arrived in the short time interval $[x, x+\Delta x]$. This means, of course, that all the remaining particles arrived after time $x$. The identity of the arriving particle can be either blue or red. Therefore,

$$
\begin{aligned}
\operatorname{Pr}\left\{\gamma^{P}=x\right\}= & \operatorname{Pr}\left\{\gamma^{P}>x\right\} \\
& \times[\operatorname{Pr}\{\text { blue arrival at } x\}+\operatorname{Pr}\{\text { red re-entrance at } x\}] .
\end{aligned}
$$


Since the arrivals of blue particles is a memoryless Poisson process with rate $\lambda_{B}$, the probability of the first blue particle to arrive during the time interval $[x, x+\Delta x]$ is approximately $\lambda_{B} \Delta x$. To compute the re-entrance probability of a red particle at time $x$, we write

$\operatorname{Pr}\{$ red re-entrance at time $x\}$

$=\int_{0}^{\infty} \operatorname{Pr}\{$ red re-entrance at time $x \mid$ the first entrance time of a red particle $=-s\}$

$\times \operatorname{Pr}\{$ a red particle first entered the simulation at time $-s\} d s$.

We consider each term factor in the integral separately. First, we recall that the conditional re-entrance time of this red particle was denoted $\gamma_{s}^{R}$ and its pdf was calculated in the previous lemma. Therefore we write

$\operatorname{Pr}\{$ red re-entrance at time $x \mid$ the first entrance time of a red particle $=-s\}$

$=\operatorname{Pr}\left\{\gamma_{s}^{R}=x \mid t_{1}=-s\right\}$.

Second, by definition,

$\operatorname{Pr}\{$ a red particle first entered the simulation at time $-s\} d s$

$=\operatorname{Pr}\{$ a blue particle entered the simulation at time $-s\} d s=\lambda_{B} d s$.

Thus, (6.10) can be rewritten as

$$
\operatorname{Pr}\left\{\gamma^{P}=x\right\}=\operatorname{Pr}\left\{\gamma^{P}>x\right\} \times \lambda_{B}\left[1+\int_{0}^{\infty} \operatorname{Pr}\left\{\gamma_{s}^{R}=x \mid t_{1}=-s\right\} d s\right] .
$$

Inserting (6.4) into (6.11), changing the order of integration in the resulting double integral, and using (6.3) and (5.2) gives

$$
\begin{aligned}
\operatorname{Pr}\left\{\gamma^{P}=x\right\} & =\lambda_{B} \operatorname{Pr}\left\{\gamma^{P}>x\right\}\left[1+\int_{0}^{\infty} f_{T}(x+u) d u \int_{u}^{\infty} \varphi(s-u) d s\right] \\
& =\lambda_{B} \operatorname{Pr}\left\{\gamma^{P}>x\right\}\left[1+\frac{F_{T}(\infty)-F_{T}(x)}{1-p}\right] \\
& =\lambda_{B} \operatorname{Pr}\left\{\gamma^{P}>x\right\} \frac{1-F_{T}(x)}{1-p} .
\end{aligned}
$$

Finally, integrating (6.12) with respect to $x$, we obtain (6.9).

Comment. The fact that the limiting PDF (6.9) is not exponential is yet another manifestation of the non-Poissonian character of the pooled process. The fact that the pooled process is not Poissonian sets this result apart from the known cases of finite mean recurrence times, as analyzed in [33], where the resulting process is Poissonian.

We now show, as mentioned in the introduction, that the pooled process is not a renewal process and has an infinite memory. Therefore its interarrival times are dependent, not identically distributed random variables. Thus, for example, the PDF of the time between the first and the second arrivals after observation begins is not 
the same as that of the time between the second and the third arrivals, and so on. The PDF of the interarrival time between the $k$ th and $k+1$ th arrivals after observation begins can be calculated as the marginal distribution of the joint distribution of the $k+1$ consecutive interarrival times.

First, we compute the PDF of the time between the first and the second arrivals of the pooled process. Applying considerations similar to the ones in the above computation of the residual first entrance time, it can be shown that

$$
\operatorname{Pr}\left\{\gamma^{P}=x \cap T_{1}^{P}>t\right\}=\Lambda F_{T}^{c}(x) F_{T}^{c}(t) \operatorname{Pr}\left\{\gamma^{P}>t+x\right\} .
$$

Thus, integrating with respect to $x$, we obtain

$$
\operatorname{Pr}\left\{T_{1}^{P}>t\right\}=\int_{0}^{\infty} \Lambda F_{T}^{c}(x) F_{T}^{c}(t) \operatorname{Pr}\left\{\gamma^{P}>t+x\right\} d x .
$$

Also, the conditional probability is given by

$$
\operatorname{Pr}\left\{T_{1}^{P}>t \mid \gamma^{P}=x\right\}=\frac{\Lambda F_{T}^{c}(x) F_{T}^{c}(t) \operatorname{Pr}\left\{\gamma^{P}>t+x\right\}}{\Lambda F_{T}^{c}(x) \operatorname{Pr}\left\{\gamma^{P}>x\right\}} .
$$

A comparison of (6.13) and (6.14) shows that the conditional PDF of $T_{1}^{P}$, given the value of the residual first entrance time $\gamma^{P}$, is different than the unconditional PDF of $T_{1}^{P}$. Therefore, the pooled process is not Poissonian, not even a renewal process, but rather has memory. Using similar methods, it is possible to show that the PDF of the $k$ th interarrival time of the pooled process depends on all previous arrival times, which means that the pooled process has infinite memory.

7. Simulation of the pooled process. In this section we present a preliminary statistical analysis of the pooled process and some computer simulation results. First, we estimate the time for convergence of a simulation of the pooled process to steady state. Then, we show both mathematically and numerically that the interarrival times of the pooled process are approximately exponentially distributed. We stress that we study only the pdf of a single interarrival time and not the joint pdf of two or more consecutive interarrival times, nor do we study the time correlations of the pooled process. These issues will be studied in a separate publication.

Consider a computer simulation of the pooled process. As analyzed in section 2 , the pooled process is the superposition of many delayed terminating renewal processes. The delayed process is the arrival process of blue particles which is Poissonian with rate $\lambda_{B}$. Thus, the arrival times of the blue particles are easily constructed by sampling their interarrival times from an exponential distribution with rate $\lambda_{B}$. For each blue particle we construct its re-entrance times into the simulation by sampling from the defective distribution $F_{T}$. Note that for each blue particle, the total number of its re-entrances follows a geometric distribution with parameter $p$. Thus, for each blue particle, this sampling procedure results in a finite sequence of random re-entrance times. The arrival times of the blue particles are formed by sorting all these entrance and re-entrance times of all particles in increasing order.

7.1. Convergence to steady state. We can estimate the rate of convergence of the pooled process to steady state, starting with no simulated particles inside the inner sphere. Specifically, we determine the minimal time $t_{S}$ at which

$$
\frac{E\left[N^{P}\left(t_{S}\right)\right]}{t_{S}}=\Lambda(1-\varepsilon) .
$$


This is a criterion for wide sense convergence to steady state [32]. An explicit expression for $E\left[N^{P}(t)\right]$ was calculated in (4.7) in terms of $\mu_{1}(s)$, the average number of re-entrances by time $s$. Since $\mu_{1}(s)$ is a monotone increasing function, it can be easily seen that $E\left[N^{P}(t)\right] / t$ is also a monotone increasing function. To find a lower bound for $t_{S}$, we use the fact that $\mu_{1}(s)$ satisfies the integral equation [30]

$$
\mu_{1}(s)=\int_{0}^{s} f_{T}(u)\left[1+\mu_{1}(s-u)\right] d u .
$$

According to (4.3), for all times $s$,

$$
\mu_{1}(s)<\mu_{1}(\infty)=\frac{p}{1-p} .
$$

Inserting this inequality into (7.2), we obtain a more refined inequality for $\mu_{1}(s)$,

$$
\mu_{1}(s)<\frac{1}{1-p} F_{T}(s)<\frac{1}{1-p} \operatorname{Pr}\left\{\tau_{\text {out }}<t\right\} .
$$

Combining (A.4) and the inequality

$$
\frac{2}{\sqrt{\pi}} \int_{x}^{\infty} e^{-u^{2}} d u \leq 1-\frac{2}{\sqrt{\pi}} x \quad \text { for } x<\frac{1}{\sqrt{2}}
$$

we obtain that

$$
\operatorname{Pr}\left\{\tau_{\text {out }}<t\right\} \leq \begin{cases}p\left(1-\frac{2}{\sqrt{\pi}} \sqrt{\frac{T_{b}}{t}}\right), & t>2 T_{b}, \\ p, & t<2 T_{b},\end{cases}
$$

where $T_{b}$ is defined in (A.5). Inserting (7.4) and (7.3) into (4.7) gives

$$
\frac{E\left[N^{P}(t)\right]}{t} \leq \Lambda\left(1-p \frac{4}{\sqrt{\pi}} \sqrt{\frac{T_{b}}{t}}+p \frac{4 \sqrt{2}}{\sqrt{\pi}} \frac{T_{b}}{t}\right) .
$$

We are now ready to apply the wide sense criteria (7.1). For $\varepsilon \ll 1, t_{S} \gg T_{b}$, so we can neglect the last term. This gives

$$
t_{S} \geq \frac{16 p^{2}}{\pi} \frac{T_{b}}{\varepsilon^{2}}
$$

To evaluate whether this time is long or short, consider just the average number of blue particle entrances during this time (neglecting their re-entrances). This number is given by the product $\lambda_{B} t_{S}$. Using (3.5) and (7.6) for $\lambda_{B}$ and $t_{S}$, respectively, gives

$$
E\left[N^{B}\left(t_{S}\right)\right] \geq \frac{12(1-p)^{2}}{\pi} \frac{N_{a}}{\varepsilon^{2}},
$$

where $N_{a}=4 / 3 \pi a^{3} \rho \gg 1$ denotes the average number of simulated particles in the inner sphere. Therefore, to obtain wide sense convergence up to one percent for a simulation with an average of $N_{a}=400$ particles in the inner sphere, the total number of blue particle entrances during this time is of the order of at least $N_{a} / \varepsilon^{2}=4,000,000$ particles. Note that in a realistic simulation, the time steps of ionic motion are much smaller than the times between consecutive entrances of particles into the simulation. Therefore, (7.7) implies that a simulation that has not started in steady state must be run a prohibitively large number of time steps until convergence to steady state is achieved. Therefore, as discussed in section 2 , an algorithm to run the simulation in steady state from the beginning is needed. 
7.2. Short interarrival times are approximately exponential. Inserting (6.9) into (6.13) gives the following expression for the distribution of $T_{1}^{P}$ :

$$
\operatorname{Pr}\left\{T_{1}^{P}>t\right\}=F_{T}^{c}(t) \int_{0}^{\infty} \Lambda F_{T}^{c}(x) e^{-\Lambda(x+t)} e^{\Lambda \int_{0}^{t+x} F_{T}(s) d s} d x .
$$

We now analyze the short time and long time behavior of this distribution. First we consider short times, $t \ll T_{b}$. In this case we make a change of variables $x=T_{b} u$ and $s=T_{b} w$ in the integrals in (7.8). This gives

$$
\operatorname{Pr}\left\{T_{1}^{P}>t\right\}=F_{T}^{c}(t) e^{-\Lambda t} \int_{0}^{\infty} \Lambda T_{b} F_{T}^{c}\left(T_{b} u\right) e^{-\Lambda T_{b}\left(u-\int_{0}^{t / T_{b}+u} F_{T}\left(T_{b} w\right) d w\right)} d u
$$

According to our assumptions,

$$
\Lambda T_{b}=\frac{3}{4} \frac{1-p}{p^{2}} N_{a} \gg 1 .
$$

Therefore, applying Laplace's method for the approximation of the integral in (7.9) gives

$$
\operatorname{Pr}\left\{T_{1}^{P}>t\right\} \approx F_{T}^{c}(t) e^{-\Lambda t} e^{\Lambda \int_{0}^{t} F_{T}(s) d s} .
$$

For short times, $t \ll T_{b}, F_{T}(t) \ll 1$, so that we have the approximation

$$
\operatorname{Pr}\left\{T_{1}^{P}>t\right\}=e^{-\Lambda t}(1+o(1)) \text { for } t \ll T_{b} .
$$

Next, we consider the long time behavior of $\operatorname{Pr}\left\{T_{1}^{P}>t\right\}$. For times $t \gg T_{b}$, we have $F_{T}(s) \approx p$, and therefore

$$
\begin{aligned}
\int_{0}^{x+t} F_{T}(s) d s & =\int_{0}^{x} F_{T}(s) d s+\int_{x}^{x+t} F_{T}(s) d s \\
& \approx \int_{0}^{x} F_{T}(s) d s+t p .
\end{aligned}
$$

Inserting (7.10) into (7.8) gives

$$
\operatorname{Pr}\left\{T_{1}^{P}>t\right\} \approx(1-p) \exp \left(-\lambda_{B} t\right) \text { for } t \gg T_{b} .
$$

To conclude, the interarrival time $T_{1}^{P}$ is approximately exponential with rate $\Lambda$ for short times, but due to possible particle recirculations its distribution has a different exponential tail with rate $\lambda_{B}<\Lambda$. Note, however, that since interarrival times are of the order of $1 / \Lambda$, and $1 / \Lambda \ll T_{b}$, all interarrival times of the pooled process are approximately exponentially distributed with rate $\Lambda$.

7.3. Simulation results. A simulation of the pooled process for uncharged particles has been run, according to the principles presented at the beginning of this section, with the typical values $a=50 \AA, r_{0}=80 \AA, D=10^{-9} \mathrm{~m}^{2} / \mathrm{sec}$, and $\rho=0.1 \mathrm{M}$. These parameters give a value $p=0.625$, for the return probability, and a value $N_{a}=31$, for the average number of simulated particles inside the inner sphere. The average interarrival time of the pooled process is $1 / \Lambda=10^{-10} \mathrm{sec}$, compared to $T_{b}=22.5 \times 10^{-10} \mathrm{sec}$, so that indeed $1 / \Lambda \ll T_{b}$. 

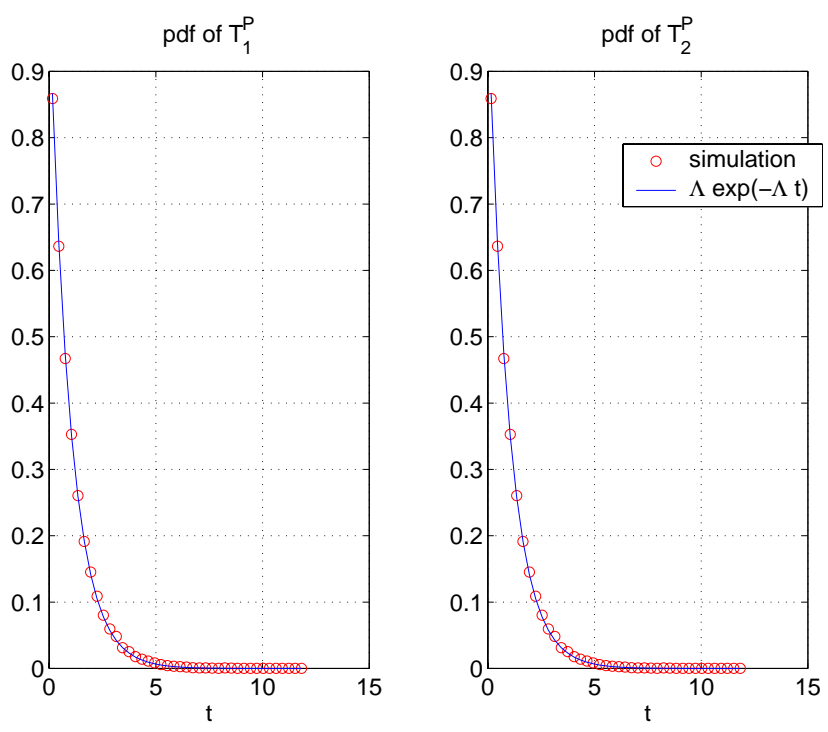

FIG. 3. The pdfs of $T_{1}^{P}$ (left) and $T_{2}^{P}$ (right), as computed from the simulation, superimposed on the exponential density with rate $\Lambda$.

In Figure 3 the graphs of the pdfs of $T_{1}^{P}$ and $T_{2}^{P}$ are superimposed on the exponential pdf with rate $\Lambda$. The pdfs of $T_{1}^{P}$ and $T_{2}^{P}$ are the result of about 250,000 samples of interarrival times of the pooled process. It is apparent that for times shorter than $T_{b}$ both $T_{1}^{P}$ and $T_{2}^{P}$ are exponentially distributed with rate $\Lambda$. In all of the samples, not even once did an interarrival time longer than $T_{b}$ occur in the simulation. Therefore, the theoretically predicted exponential tail with rate $\lambda_{B}$ cannot be observed in these graphs. Finally, we note that $T_{1}^{P}$ and $T_{2}^{P}$ are dependent, with a correlation coefficient $r=\operatorname{cov}\left(T_{1}^{P}, T_{2}^{P}\right) / \sigma_{1} \sigma_{2} \approx-0.001$. The fact that the correlation coefficient is negative is not surprising. It reflects the higher probability of recirculation in $T_{2}^{P}$ when $T_{1}^{P}$ is large. This means that when $T_{1}^{P}$ is long there is a higher probability that $T_{2}^{P}$ is short. The small correlation coefficient between $T_{1}^{P}$ and $T_{2}^{P}$ is a consequence of the short interarrival times of the pooled process, $1 / \Lambda$, relative to the characteristic time for recirculation, $T_{b}$. A detailed analytical and numerical study of the statistical properties of the pooled process will be done elsewhere.

8. Summary and discussion. The time course of the exchange of ions between a test volume embedded in a continuum with a buffer region has been studied. The study of this time course is the basis for a simulation of uncharged and charged particles in a solution. The process of injecting new particles into the simulation has been identified as a stationary pooled process composed of an infinite number of delayed terminating independent renewal processes. While the pooled process converges to a stationary steady state, it is neither a renewal nor a Markov process. We have calculated the first two moments of this process, as well as the probability distribution of its residual time, and the joint distribution of the residual and the next arrival. From these calculations, it is apparent that the pooled process has an infinite memory. Therefore, to run the exact time course of this process in steady state, an infinite record of all past entrances and exits needs to be kept. To avoid this complexity, we have found that under some conditions on the size of the simulation 
and buffer regions the pooled process can be approximated by a memoryless Poisson process. This approximation retains the average influx of the pooled process, but underestimates its variance. Our analysis shows how the parameters $a$ and $r_{0}$ control the size of the simulation and the accuracy of our proposed approximation.

A closely related mathematical problem is considered in [30, chap. 5, sect. 9], where the renewal periods are assumed to have finite moments, in contrast with the case at hand in which the renewal periods may be infinite with positive probability. In [30], the case of infinitely many uniform sparse renewal processes is considered, and it is shown that under certain conditions on the sparseness, the pooled process becomes Poissonian as the number of processes increases to infinity. As we have seen, in our case the resulting process in not Poissonian.

The application of our results to an actual simulation is different for charged or uncharged particles. The approximations that we derived are not necessary for a simulation of uncharged Brownian particles, though they are necessary for a simulation of charged particles, as discussed below. For uncharged particles the stationary pooled process can be constructed offline to provide the random injection times of particles into the simulation. Such a pooled process has to be constructed for each choice of the parameters $\lambda_{B}$ and $p$, that is, for each set of values for the parameters $D, \rho, a$, and $r_{0}$. With the correct choice of injection times, such a simulation reaches steady state immediately. If a wrong injection time course is adopted, there is a depletion or overcrowding of particles in the simulation region, which renders the simulation not self-consistent.

The simulation of charged particles is completely different from that of uncharged particles. In the uncharged case the mean field is always zero and thus the densities of all species in the bath remain constant throughout the time course of the simulation. In contrast, charged particles cause fluctuations in the net charge inside the simulation volume. The nonzero net charge creates a nonvanishing time dependent electrostatic field outside the simulation region that affects the continuum densities in the bath near the simulation region. Thus, if the net charge in the simulation region is positive, the bath density of positive charges decreases and the density of the negative charges increases in the neighborhood around the simulation region. These changes, in turn, affect the effective entrance rates of the different species into the simulation. Therefore, for charged particles, each configuration of net charge in the simulation region requires the construction of a new table of the pooled process suitable for it. Specifically, each entrance or exit changes the net charge inside the simulation region, which, in turn, changes the entrance rates into the simulation region.

Under the assumption of a fast bath, ${ }^{1}$ the entrance law of a new ion into the simulation is that of the residual of the pooled process (6.9) that corresponds to the instantaneous concentrations due to the momentary net charge inside the simulation. These conditions change every time an ion enters or exits the simulation. Our analytical expression for the PDF of the residual time eliminates the need to run a simulation of the pooled process (as described in section 7) every time conditions change. In this way, a small portion of the bulk solution can be studied without the need to resort to ad hoc assumptions, such as artificial periodic boundary conditions.

In our model and analysis of a simulation of interacting ions (e.g., charged ions or ions with finite volume), we have used implicitly the concept of a self-consistent simulation. This notion is concerned with the detailed laws of ionic motion in the

\footnotetext{
${ }^{1}$ This means that the time to equilibrate the densities in the bath is shorter than the time between changes in the net charge inside the simulation region.
} 
simulation region and the effective motion of ions in the surrounding continuum. The effective diffusive motion of ions is the result of their thermal interaction with the surrounding solvent and their interactions with each other. Thus, the motion inside the simulation volume is governed by both an unknown diffusion coefficient of ions in infinitely dilute solution and the interionic forces (computed by the simulation). On a sufficiently coarse time scale, the resulting motion of a simulated ion can be viewed as an effective diffusion with an effective diffusion coefficient that can be calculated from statistics of simulated trajectories. This calculated effective diffusion coefficient must be equal to the assumed (experimentally measured) diffusion coefficient in the bulk solution. This is a self-consistency requirement from the simulation. In addition, the average concentrations of the ionic species inside the simulation volume must be the same as those assumed in the bulk solution, as mentioned above. This is another self-consistency requirement. Still another self-consistency requirement is concerned with the notion of chemical activity. The concentrations of ions in the presence of an electrostatic field is different than that in the absence of a field, as can be readily seen from the Poisson-Boltzmann theory [12]. To compensate for the replacement of charged particles with independent uncharged particles the notion of activity has been introduced in physical chemistry [12]. More precisely, the change in the chemical potential as a function of particle density is assumed to take a simple form derived from the theory of gases, which replaces the physical density with a larger effective density. The ratio of the two densities is the activity factor. It is a directly measurable physical parameter. In a self-consistent simulation of charged particles, the unknown activity and diffusion coefficients in an infinitely dilute solution have to be chosen in such a way that all the above-mentioned self-consistency conditions are met. Finally, in addition to these self-consistency conditions, the electrostatic field has to be calculated in a self-consistent way at each time step of the simulation. A detailed description of a self-consistent simulation of charged particles will be presented in a separate paper. If the correct time course of the simulation is not followed it may be difficult to meet some of these self-consistency requirements.

Simulations of ions in solution have a wide range of applications. An important one is the theoretical study of permeation of uncharged molecules and ions through protein channels of biological membranes [34], [35]. Protein channels are small natural nano-devices of length in the range of $20-100 \AA$, and 5-20 $\AA$ diameter. A computer simulation of a channel involves the simulation of the mobile ions both inside the channel and around it, in a volume comparable to the channel size. In the spirit of the theory discussed in this paper, such a simulation must be connected to its surrounding continuum. This leads to a small simulation with large time dependent density and potential fluctuations. The results of this paper are directly applicable to a simulation of permeation of uncharged molecules through protein channels, such as maltoporins that conduct sugar. Although the motion of the sugar molecules inside the maltoporin channel cannot be assumed a diffusion process and has to be simulated by molecular dynamics, the connection of the simulation to the continuum is described by the present work. For channels that conduct ions or other charged molecules, there are additional new elements in their simulation, namely, the presence of an impermeable membrane and a permanent charge profile of the channel itself, inside the simulation volume. The injection process of new particles into the simulation region on both sides of the membrane is similar to that described above. There are many differences, though, between a simulation with and without a channel, that are a subject for a separate study. 
Appendix A. The time distribution outside the simulation. We now compute the distribution of $\tau_{\text {out }}$, the time a nonsimulated (green) particle spends outside the simulation until its next re-entrance into the simulation. To this end, we denote by $p\left(r, t \mid r_{0}\right)$ the (radial) conditional probability density of the particle's location at time $t$, given that it has exited the simulation at the outer sphere at time $t=0$ and has not yet returned to the inner sphere. Obviously, in terms of this distribution,

$$
\operatorname{Pr}\left\{\tau_{\text {out }}>t\right\}=\int_{a}^{\infty} 4 \pi r^{2} p_{\text {out }}\left(r, t \mid r_{0}\right) d r .
$$

According to our assumptions, nonsimulated green particles perform independent free Brownian motion with diffusion coefficient $D$. Thus, the pdf $p_{\text {out }}\left(r, t \mid r_{0}\right)$ is the solution of the Fokker-Planck equation [31]

$$
\begin{aligned}
\frac{\partial}{\partial t} p_{\text {out }}\left(r, t \mid r_{0}\right) & =D \Delta p\left(r, t \mid r_{0}\right), \quad a<r<\infty, \\
p_{\text {out }}\left(a, t \mid r_{0}\right) & =0, \quad t>0, \\
p_{\text {out }}\left(r, 0 \mid r_{0}\right) & =\frac{\delta\left(r-r_{0}\right)}{4 \pi r_{0}^{2}}, \quad a<r<\infty .
\end{aligned}
$$

The solution of (A.2) is given by

$$
p_{\text {out }}\left(r, t \mid r_{0}\right)=\frac{1}{(4 \pi D t)^{1 / 2}} \frac{1}{4 \pi r_{0}} \frac{1}{r}\left\{e^{-\left(r-r_{0}\right)^{2} / 4 D t}-e^{-\left(r+r_{0}-2 a\right)^{2} / 4 D t}\right\} .
$$

Inserting (A.3) into (A.1) gives

$$
\operatorname{Pr}\left\{\tau_{\text {out }} \leq t \mid r_{0}\right\}=p \frac{2}{\sqrt{\pi}} \int_{\sqrt{T_{b} / t}}^{\infty} e^{-u^{2}} d u,
$$

where

$$
T_{b}=\frac{\left(r_{0}-a\right)^{2}}{4 D}
$$

is a characteristic time for the motion of a particle from the outer sphere to reach the inner sphere.

Equation (A.4) shows that $\tau_{\text {out }}$ can be infinite with probability $1-p>0$, that is, it has a defective probability distribution. It follows that it has an infinite mean value. The pdf of its defective distribution, given by

$$
f_{\tau_{\text {out }}}(t)=\frac{d}{d t} \operatorname{Pr}\left\{\tau_{\text {out }} \leq t \mid r_{0}\right\}=p \frac{1}{\sqrt{\pi}} \frac{\sqrt{T_{b}}}{t^{3 / 2}} \exp \left\{-\frac{T_{b}}{t}\right\}
$$

gives

$$
\int_{0}^{\infty} t f_{\tau_{\text {out }}}(t) d t=\infty
$$

Moreover, we have the following proposition. 
Proposition A.1. The first moment of $\tau_{\text {out }}$, conditioned on $\left\{\tau_{\text {out }}<\infty\right\}$, is infinite.

Proof. Since

$$
\operatorname{Pr}\left\{\tau_{\text {out }}<t \mid \tau_{\text {out }}<\infty\right\}=\frac{\operatorname{Pr}\left\{\tau_{\text {out }}<t, \tau_{\text {out }}<\infty\right\}}{\operatorname{Pr}\left\{\tau_{\text {out }}<\infty\right\}}=\frac{\operatorname{Pr}\left\{\tau_{\text {out }}<t\right\}}{p},
$$

we obtain, in view of (A.6), that

$$
\int_{0}^{\infty} t d \operatorname{Pr}\left\{\tau_{\text {out }}<t \mid \tau_{\text {out }}<\infty\right\}=\frac{1}{\sqrt{\pi}} \int_{0}^{\infty} \frac{\sqrt{T_{b}}}{t^{1 / 2}} \exp \left\{-\frac{T_{b}}{t}\right\} d t=\infty
$$

\section{REFERENCES}

[1] J. P. Valleau and S. G. Whittington, A guide to Monte Carlo for statistical mechanics: 1. Highways, in Statistical Mechanics: Part A: Equilibrium Techniques, Modern Theoretical Chemistry 5, B. J. Berbe, ed., Plenum Press, New York, 1977, p. 15.

[2] G. King And A. WARshel, A surface constraint all atom solvent model for effective simulations of polar solutions, J. Chem. Phys., 91 (1989), p. 3647.

[3] J. M. Caillol, D. Levesque, and J. J. Weiss, Electrical properties of polarizable ionic solutions II: Computer simulation results, J. Chem. Phys., 91 (1989), pp. 5555-5566.

[4] J. M. CAILlol, A new potential for the numerical simulations of electrolyte solutions on a hyper-sphere, J. Chem. Phys., 99 (1993), pp. 8953-8963.

[5] Y.-Y. Shamm And A. WARshel, The surface constraint all atom model provides size independent results in calculations hydration free energies, J. Chem. Phys., 109 (1998), pp. 79407944.

[6] G. Ciccotti And A. Tenenbaum, Canonical ensemble and nonequilibrium states by molecular dynamics, J. Statist. Phys., 23 (1980), p. 767.

[7] M. Berkowitz and J. A. MCCammon, Molecular dynamics with stochastic boundary conditions, Chem. Phys. Lett., 90 (1982), pp. 215-217.

[8] C. L Brooks III and M. Karplus, Deformable stochastic boundaries in molecular dynamics, J. Chem. Phys., 79 (1983), p. 6312.

[9] A. C. Belch And M. Berkowitz, Molecular dynamics simulations of TIPS2 water restricted by a spherical hydrophobic boundary, Chem. Phys. Lett., 113 (1985), pp. 278-282.

[10] I. M. Wonpil, S. Seefeld, And B. Roux, A grand canonical Monte Carlo-Brownian dynamics algorithm for simulating ion channel, Biophys. J., 79 (2000), p. 788-801.

[11] S. Chandrasekhar, Stochastic problems in physics and astronomy, Rev. Mod. Phys., 15 (1943), pp. 1-89.

[12] R. S. Berry, S. Rice, And J. Ross, Physical Chemistry, 2nd ed., Oxford University Press, Oxford, 2000.

[13] S. G. Brush, The Kind of Motion We Call Heat, Vol. I, North-Holland, Amsterdam, 1986.

[14] S. G. Brush, The Kind of Motion We Call Heat, Vol. II, North-Holland, Amsterdam, 1986.

[15] M. P. Allen and D. J. Tildesley, Computer Simulation of Liquids, Oxford University Press, Oxford, 1991.

[16] B. Corry, S. Kuyucak, And S. Chung, Tests of continuum theories as models of ion channels. II. Poisson-Nernst-Planck theory versus Brownian dynamics, Biophys. J., 78 (2000), pp. 2364-2381.

[17] F. L. Roman, J. A. White, and S. Velasco, Fluctuations in an equilibrium hard-disk fluid: Explicit size effects, J. Chem. Phys., 107 (1997), pp. 4635-4641.

[18] F. L. Roman, J. A. White, and S. Velasco, Fluctuations in the number of particles of the ideal gas: A simple example of explicit finite-size effects, Amer. J. Phys., 67 (1999), pp. 1149-1151.

[19] J. Horbach, W. Kob, K. Binder, and C. A. Angell, Finite size effects in simulations of glass dynamics, Phys. Rev. E., 54 (1996), pp. 5897-5900.

[20] T. M. Nymand AND P. Linse, Ewald summation and reaction field methods for potentials with atomic charges, dipoles, and polarizabilities, J. Chem. Phys., 112 (2000), pp. 6152-6160.

[21] J. M. CAillol, A Monte Carlo study of the dielectric constant of the restricted primitive model of electrolytes on the vapor branch of the coexistence line, J. Chem. Phys., 102 (1995), pp. 5471-5479. 
[22] R. A. Friedman And M. Mezei, The potentials of mean force of sodium chloride and sodium dimethylphosphate in water: An application of adaptive umbrella sampling, J. Chem. Phys., 102 (1995), pp. 419-426.

[23] M. P. Allen and D. J. Tildesley, eds., Computer Simulation in Chemical Physics, NATO ASI Ser. C Math. Phys. Sci. 397, Kluwer Academic Publishers, Dordrecht, The Netherlands, 1992.

[24] B. Nadler, T. NAeH, And Z. Schuss, The stationary arrival process of independent diffusers from a continuum to an absorbing boundary is Poissonian, SIAM J. Appl. Math., 62 (2001), pp. 433-447.

[25] R. VON SMOLUCHOWSKI, Versuch einer mathematischen Theorie der Koagulationskinetic koloider Lösungen, Zeits. Phys. Chem., 92 (1917), p. 129.

[26] P. BordewiJk, Defect-diffusion models of dielectric relaxation, Chem. Phys. Lett., 32 (1975), pp. 592-596.

[27] T. NAeH-Galor, Simulation of Ionic Solution, Ph.D. dissertation, Tel-Aviv University, Tel Aviv, Israel, 2001.

[28] W. FelLer, An Introduction to Probability Theory and Its Applications, Vol. 2, John Wiley, New York, 1970.

[29] B. NADler, Density Fluctuations, M.Sc. dissertation, Tel-Aviv University, Tel Aviv, Israel, 1994.

[30] S. Karlin and H. M. TaYlor, A First Course in Stochastic Processes, Academic Press, New York, 1975.

[31] Z. Schuss, Theory and Application of Stochastic Differential Equation, John Wiley, New York, 1980.

[32] A. Papoulis, Probability, Random Variables and Stochastic Processes, 3rd ed., McGraw-Hill, New York, Toronto, London, 1991.

[33] D. R. Cox And P. A. W. Lewis, The Statistical Analysis of Series of Events, Methuen, London, 1968.

[34] B. Hille, Ionic Channels of Excitable Membranes, 2nd ed., Sinauer Associates, Sutherland, MA, 1992.

[35] R. S. Eisenberg, From structure to function in open ionic channels, J. Mem. Biol., 171 (1999), pp. $1-24$. 